\title{
Einsatz von Opioidantagonisten in der abstinenzerhaltenden Behandlung der Opiatabhängigkeit
}

Opioid-Antagonists in the Abstinence Orientated Treatment of Opiate Addiction

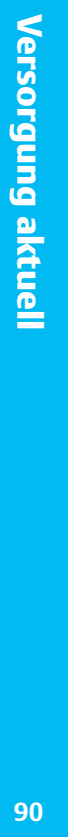

\section{Zusammenfassung}

Die Behandlung der Opiatabhängigkeit mit Opioidantagonisten ist eine theoretisch viel versprechende, in der Praxis aber vergleichsweise selten genutzte Therapieoption. Die Übersichtsarbeit fasst vorliegende Studien zur Behandlung mit Opiatantagonisten, insbesondere Naltrexon, zusammen. Die vorliegenden Therapieergebnisse sind insgesamt positiv zu bewerten, wobei spezifische Indikationskriterien für den praktischen Einsatz und die Notwendigkeit einer begleitenden psychosozialen Behandlung zu berücksichtigen sind. So können Opiatantagonisten als medikamentöser Teil eines therapeutischen Gesamtkonzepts wesentlich dazu beitragen, Abstinenz von Opiaten langfristig zu erhalten.

Schliuisselwörter

Opioid $\cdot$ Opiat $\cdot$ Therapie $\cdot$ Sucht $\cdot$ Naltrexon

\section{Abstract}

Treatment of subjects addicted to opiates with opioid-antagonists is a theoretically promising option; however, it is rather seldom included in general therapeutic intervention in clinical practice. This review now summarizes results of studies on treatment with opioid antagonists, especially naltrexone, and suggests general guidelines for the use of opioid antagonists. Especially the inclusion of psychosocial interventions in addition to pharmacotherapy seems to be a main condition for positive treatment outcome. In general, opioid-antagonists are an important part of a multi-modal treatment programme to support opiate addicts maintaining long-term abstinence.

\section{Key words}

Opioid $\cdot$ opiate $\cdot$ treatment $\cdot$ addiction $\cdot$ naltrexone

\section{Einleitung}

Die Behandlung der Opiatabhängigkeit mit Opioidantagonisten ist eine theoretisch viel versprechende, in der Praxis aber vergleichsweise selten genutzte Therapieoption. Als Gründe hierfür können einerseits generelle Akzeptanzprobleme auf Seiten der Patienten genannt werden, andererseits aber auch Unsicherheiten auf Behandlerseite bezüglich der korrekten Indikationsstellung, der praktischen Therapieplanung und nicht zuletzt bezüglich der Interpretation der vorliegenden zum Teil widersprüchlichen Wirksamkeits- und Verträglichkeitsdaten.
Dennoch liegt eine Reihe von Befunden vor, die nachweisen, dass Opiatantagonisten als medikamentöser Teil eines therapeutischen Gesamtkonzepts wesentlich dazu beitragen können, Abstinenz von Opiaten langfristig zu erhalten. Zur pharmakotherapeutischen Abstinenzunterstützung für den Langzeiteinsatz bei Opiatabhängigen ist in Deutschland bislang allein Naltrexon $\left(\right.$ Nemexin $\left.^{\circledR}\right)$ zugelassen. Voraussetzungen für dessen Einsatz sind eine abgeschlossene Entzugsbehandlung und eine parallel in Anspruch genommene psychosoziale Therapie [1].

Institutsangaben

Klinik für Psychiatrie und Psychotherapie, Universitätsklinikum Hamburg-Eppendorf

Korrespondenzadresse

PD Dr. med. Falk Kiefer · Klinik für Psychiatrie und Psychotherapie Universitätsklinikum Hamburg-Eppendorf .

Martinistr. 52 20251 Hamburg · E-mail: E-mail: kiefer@uke.uni-hamburg.de

Bibliografie

Suchttherapie 2004; 5: 90-93 @ Georg Thieme Verlag KG Stuttgart · New York

DOI 10.1055/s-2004-813163

ISSN 1439-9903 
Befunde zur Pharmakologie, klinische Wirksamkeitsdaten und hierauf beruhende Behandlungsempfehlungen sind in der vorliegenden Übersichtsarbeit zusammengefasst.

\section{Pharmakologie}

Naltrexon, das als nahezu reiner Opiatantagonist präferenziell am $\mu$-Rezeptor wirkt, ist ohne klinisch signifikante Eigenwirkung und erzeugt weder Toleranz noch Abhängigkeit [2, 3]. Es verdrängt kompetitiv Opiate von den Bindungsstellen; bei Einnahme ist selbst nach Applikation hoher Opiatdosen eine Opiatwirkung nicht zu beobachten $[4,5]$.

Die Vorteile von Naltrexon im klinischen Einsatz liegen in seiner hohen Affinität zu den Rezeptorbindungsstellen und der langen Wirkdauer: Nach Einnahme von $50 \mathrm{mg}$ werden sämtliche Opiatwirkungen über 24 Std., bei $100 \mathrm{mg}$ über $48 \mathrm{Std}$. und bei $150 \mathrm{mg}$ über 72 Std. blockiert [6, 7]. An der lang anhaltenden Wirkung ist ebenfalls der Metabolit $\beta$-Naltrexol beteiligt. Das Nebenwirkungsprofil ist in der klinischen Behandlung sehr günstig (Übersicht: [5]). Vornehmlich werden zu Beginn der Behandlung gastrointestinale Beschwerden, Appetitminderung und selten allergische Reaktionen beschrieben. Eine merkliche Anhedonie, wie sie von vielen Patienten befürchtet wird, tritt in der Regel nicht auf. Selbst bei Dosen von $300 \mathrm{mg} / \mathrm{d}$ ließen sich keine unerwünschten Wirkungen auf Stimmung und kognitive Funktionen nachweisen. Ausschlusskriterien für die Behandlung mit Naltrexon sind akute oder schwere chronische Hepatitiden.

\section{Klinische Studien}

Basierend auf der Konditionierungstheorie von Wikler [8 - 11], gab es in den siebziger Jahren erste Versuche, durch Blockade der Opiatwirkung (als „medikamentöser Schutz“ nach der Opiatentgiftung) die Voraussetzung zur Erlernung suchtfreier Denk- und Verhaltensweisen zu schaffen. Untersuchungen in den Vereinigten Staaten und in Europa mit Naltrexon in der Rückfallprophylaxe bei Opiatabhängigkeit ergaben anfangs sehr differierende Ergebnisse [12 - 24]. Insbesondere erste Studien erbrachten im Hinblick auf die eingehaltenen Abstinenzperioden eher enttäuschende Resultate. Spätere Arbeiten wiesen dagegen positivere Resultate auf, wobei hierbei insbesondere Lopez Ibor et al. [25] und Ochoa et al. $[26,27]$ zu nennen sind, die Abstinenzraten von im Mittel 51\% nach 1 Behandlungsjahr erzielten. Doppelblinde, placebokontrollierte Studien ergaben weitere positive Ergebnisse in Bezug auf die Verlängerung der Abstinenzrate [28 - 32] bzw. die Reduktion des Suchtdrucks (des „Craving“ [32, 33]). Obwohl man davon ausgehen muss, dass Patienten nach Opiatzufuhr das Vorhandensein bzw. Fehlen einer Opiatrezeptorblockade sehr leicht selbst feststellen können, zeigte sich interessanterweise in einer Untersuchung, dass auch bei einigen Placebo-Patienten eine Heroineinnahme subjektiv „nicht wirkte“ [30].

Eine Erklärung für die frühen, insgesamt negativen Studienresultate beruht auf methodologischen Spezifikationen, die vornehmlich in einer damals für die Methadonbehandlung geltenden klinischen Praxis begründet waren. Die Abgabe von Naltrexon erfolgte ohne zureichende psychotherapeutische und psycho- soziale Unterstützung, der therapeutische Schwerpunkt wurde in der Verabreichung des Opiatantagonisten gesehen.

\section{Klinischer Einsatz}

Bereits Kleber [33, 34] und Kosten [35] empfahlen in ihren Übersichtsartikeln eine differenzierte Anwendung von Naltrexon. Heute muss die Therapie mit Naltrexon als Teil eines individuellen therapeutischen Gesamtkonzepts gesehen werden $[13,14$, 36]. Nicht jeder Patient ist für eine Naltrexon-Behandlung geeignet. So stellten Koc und Poser [37], die Naltrexon im Rahmen der Göttinger Methadonstudie einsetzten, fest, dass die hohe Abbruchquote durch die anfänglich sehr breite Indikationsstellung bedingt war. Sie stellten eine Liste mit Merkmalen auf, die sich als prognostisch günstig erwiesen: Der Patient sollte eine hohe Abstinenzmotivaton mitbringen und nicht mehr als 5 Jahre abhängig sein, d.h. sich im Frühstadium der Opiatabhängigkeit befinden. Eine vorhandene oder wieder herstellbare soziale Integration (Beruf, Partnerschaft, Familie, Wohnung) wurde ebenso als Voraussetzung für eine erfolgreiche Behandlung gesehen wie eine tragfähige Beziehung zum Therapeuten und die Identifikation des Patienten mit dem Therapieprinzip. Diese prognostisch günstigen Auswahlkriterien sind auch durch andere Autoren beschrieben worden, die hohe Abstinenzraten mit gezielter Patientenselektion erreichten [38-41].

Vor Einsatz der Medikation muss eine Aufklärung erfolgen und sichergestellt werden, dass der Patient frei von Opiaten ist, um ein plötzliches und massiv auftretendes Opiatentzugssyndrom zu vermeiden. Bei Heroinabhängigkeit sollte ein zeitlicher Abstand zur letzten Dosis von sechs Tagen, bei Methadonabhängigkeit von zehn Tagen eingehalten werden. Eine Urinprobe zum Nachweis von Opiaten muss direkt vor Beginn der Naltrexon-Behandlung negativ ausfallen. Zur Absicherung kann ein Test mit einer Ampulle des kurz wirksamen Opiatantagonisten Naloxon (Narcanti ${ }^{\circledR}$ ) i. v. erfolgen. Kommt es dabei trotz vorheriger Absicherung zur Entzugssymptomatik, so kann diese durch Gabe von Clonidin (Catapresan ${ }^{\circledR}$ ) abgemildert werden.

Falls kein Naloxon-Test vorgenommen wird, sollte der Patient nach der ersten Naltrexon-Gabe über zwei Stunden unter Beobachtung verbleiben.

Die Einnahme kann durch den Patienten selbst (meist $50 \mathrm{mg} / \mathrm{d}=$ 1 Tablette) oder kontrolliert durch Arzt oder Pflegepersonal erfolgen (meist $3 \times /$ Woche: Montag und Mittwoch je $100 \mathrm{mg}$, Freitag $150 \mathrm{mg}$ ). Letzteres ist insbesondere für die ambulante Therapie praktikabel und durch die lang anhaltende Wirksamkeit von Naltrexon ermöglicht. Eine Einnahme von Opioiden unter NaltrexonBehandlung führt aufgrund des Ausbleibens einer merklichen Opiatwirkung nicht zu einem progredienten Rückfall.

Unter der Behandlung mit Naltrexon kommt es bereits innerhalb von 2-3 Wochen durch die Heraufregulierung der Dichte und Affinität von Opiatrezeptoren zu einem „Toleranzverlust“, d.h. zu einer erhöhten Empfindlichkeit gegenüber Opioiden. Patienten müssen hierüber aufgeklärt werden, da bei Abbruch der Behandlung die Zufuhr der für den Patienten vorher „normalen“ Opiatdosis tödliche Folgen haben kann. 
Untersuchungen zur „optimalen Behandlungsdauer“ mit Naltrexon existieren bisher nicht, aufgrund der lang anhaltenden psychischen Abhängigkeit sollte eine erfolgreiche Behandlung nicht vor Ablauf von 3 Monaten beendet werden, in einigen Fällen wurde sie länger als 1 Jahr erfolgreich durchgeführt [37]. Naltrexon kann auch der Überbrückung nach Opiatentzug bis zum Antritt einer stationären Langzeitentwöhnungstherapie oder im Anschluss daran dienen. Nach Abschluss einer stationären Therapie eignet sich Naltrexon zum Einsatz bei der ambulanten poststationären Nachbetreuung und länger dauernder ambulanter Behandlung insbesondere bei Patienten mit Suchtdruck, Rückfallangst und Wunsch auf medikamentöse Sicherheit in Phasen hoher Rückfallgefährdung [30].

Die Behandlung mit Naltrexon ist am besten für monovalent Opiatabhängige geeignet, da bei politoxikomanen Patienten die Wirkung der anderen Suchtstoffe nicht beeinflusst wird und die Gefahr einer Suchtverlagerung gegeben ist. Aufgrund der zentralen Bedeutung der Opioide für die Steuerung des endogenen „Belohnungssystems“ gibt es aber Hinweise darauf, dass Naltrexon allgemein die subjektiv positiven Effekte von Suchtdrogen reduziert.

\section{Diskussion}

Der Opioidantagonist Naltrexon kann dazu beitragen, die Wahrscheinlichkeit eines Rückfalls bei Opiatabhängigkeit zu mindern und hierdurch Sicherheit und in der Folge Selbstwirksamkeitserwartung zu vermitteln. Die Behandlung ermöglicht, die gesicherte Abstinenz zur Inanspruchnahme weitergehender soziound psychotherapeutischer Maßnahmen zu nutzen. Phasen der Labilität und der Rückfallgefährdung können darüber hinaus auch nach länger dauernder Abstinenz überbrückt werden. Entgegen früherer Vorbehalte kann die Einnahme einer rückfallprophylaktischen Medikation auch zur therapeutisch wertvollen Auseinandersetzung mit dem eigenen Suchtverhalten beitragen. Der Patient entscheidet sich aktiv im Vorfeld, eine wirksame Drogeneinnahme im Sinne eines Vermeidungsverhaltens während der Behandlung auszuschließen. Hierdurch kann eine direktere Auseinandersetzung mit der Abhängigkeitserkrankung, mit assoziierten Beziehungsmustern und Verhaltensweisen erleichtert werden [28]. Therapieergebnisse kontrollierter klinischer Studien zeigen die Wirksamkeit dieses Therapieansatzes. Als „Mosaikstein“ in der Behandlung von Drogenabhängigen kann Naltrexon als medikamentöser Teil eines therapeutischen Gesamtkonzepts helfen, den Therapieerfolg langfristig zu sichern.

\section{Literatur}

${ }^{1}$ Fachinformation Nemexin ${ }^{\circledR}$ DuPont Pharma GmbH

2 Blumberg H. Analgesic properties of the narcotic antagonist EN-2234 A. Pharmacologists 1968; 10: 201

${ }^{3}$ Kleber HD, Kosten TR, Gaspari J et al. Nontolerance to the opioid antagonism of naltrexone. Biological Psychiatry 1985; 20: 66-72

${ }^{4}$ Ionescu F, Klee W, Katz R. Antagonist potency and receptor binding. Life Sciences 1975; 16: 1973-1974

${ }^{5}$ Gonzalez JP, Brogden RN. Naltrexone: A Review of its Pharmacodynamic and Pharmacokinetic Properties and Therapeutic Efficacy in the Management of Opioid Dependence. Drugs 1988; 35: 192 - 213
${ }^{6}$ Kleber HD. Final report: Double-blind, placebo controlled study administered by the National Academy of Sciences, to evaluate the safety and efficacy of the narcotic antagonist unpublished.

7 Verebey K, Volavka J, Mule SJ et al. Naltrexone: Disposition, metabolism and effects after acute and chronic dosing. Clin Pharmacol Ther 1976; $20: 315-328$

${ }^{8}$ Wikler A. Recent progress in research on the neurophysiological basis of morphine addiction. Am J Psychiatry 1948; 105: 329-338

${ }^{9}$ Wikler A. Present status of the concept of drug dependence. Psychol Med 1971; 1: 377-380

${ }^{10}$ Wikler A. Dynamics of Drug Dependence. Arch Gen Psychiatry 1973; 28: $611-616$

${ }^{11}$ Wikler A. The theoretical basis of narcotic addiction treatment with narcotic antagonists. In: Julius D, Renault P. (eds). Narcotic Antagonist: naltrexone. National Institute on Drug Abuse (NIDA) Research Monograph 9. Rockville: Government Printing Office, 1976: 119-122

${ }^{12}$ Bradford HA. Interim report on clinic intake and safety data collected from 17 NIDA funded naltrexone centers. In: Julius D, Renault P (eds). Narcotic antagonists: naltrexone. Rockville: NIDA Research Monogramm 1975: 9: 163 - 171

${ }^{13}$ Callahan EJ. Comparison of two naltrexone programmes: naltrexone alone versus naltrexone plus behaviour therapy. In: Julius D, Renault P (eds). Narcotic antagonists: naltrexone. NIDA Research Monograph. Washington DC: Supt of DocsUS Govt Print Off, 1976: 9: 150-157

${ }^{14}$ Callahan EJ, Rawson RA, McCleave B et al. The treatment of heroin addiction: Naltrexone alone Int J Addict 1980; 15: 795 - 807

${ }^{15}$ Capone TA. Psychoanalytic and behavioral considerations in antagonist and methadone programs. J Contemp Psychother 1978; 9 (2): $139-150$

${ }^{16}$ Fram DH, Marmo J, Holden R. Naltrexone treatment - the problem of patient acceptance. J Subst Abuse Treat 1989; 6: 119-122

17 Garcia-Alonso F, Gutierrez M, San L et al. A multicenter study to introduce naltrexone for opiate dependence in Spain. Drug Alcohol Depend 1989; 23 (2): $117-121$

${ }^{18}$ Greenstein RA, Arndt IC, McLellan AT et al. Naltrexone: A clinical perspective. J Clin Psychiatry 1984; 45: 25 - 28

19 O'Brien CP, Greenstein RA, Mintz J et al. Clinical experience with naltrexone. Am J Alcohol Abuse 1975; 2: 365 - 377

${ }^{20}$ Rawson RA. Naltrexone and behavior therapy for heroin addiction. In: Krasnegor NA (ed). NIDA Research Monograph 25, US Dept of Health and Human Services. Rockville, 1979: 26-43

${ }^{21}$ Resnick RB, Volavka J, Freedman AM et al. Studies of EN-1639A (naltrexone): A new narcotic antagonist. Am J Psychiatry 1974; 131: $646-650$

${ }^{22}$ Resnick RB, Schuyten-Resnick E, Washton AM. Narcotic antagonists in the treatment of opioid dependence: review and commentary. Compr Psychiatry 1979; 20: $116-125$

${ }^{23}$ Sideroff SI, Charuvastra VC, Jarvik ME. Craving in heroin addicts maintained on the opiate antagonist naltrexone. Am J Drug Alcohol Abuse 1979; 5: 415 - 423

24 Tennant FS Jr, Rawson RA, Cohen AJ et al. Clinical experience with naltrexone in suburban opioid addicts. J Clin Psychiatry 1984; 45: 42 - 45

${ }^{25}$ Lopez-Ibor JL. The medical model in the management of heroin dependence. In: Stefanies CN, Rabavilas AD, Soldatos CR. (eds). Classification and Psychopathology; Child Psychiatry; Substance Use. Proc 8th World Congr Psychiatry, Athens, 12 -19 October 1989, Vol 1. Amsterdam Excerpta Medica, 1990

${ }^{26}$ Ochoa E, Arias F, Somoza JC et al. Treatment with naltrexone in opiate dependents: 2 years' follow-up. Actas Luso Esp Neurol Psiquiatr 1992; $20(5): 215-229$

27 Ochoa E, Arias F, Somoza JC et al. Tratamiento con naltrexona en dependientes de opiáceos: dos anos y medio de seguimiento. Arch Neurobiol 1992; 55 (5): $224-227$

${ }^{28}$ Hollister LE. Clinical evaluation of naltrexone treatment of opiate-dependent individuals. Report on the National Research Council Committee on Clinical Evaluation of Narcotic Antagonists. Arch Gen Psychiatry 1978; 35 : $335-340$

${ }^{29}$ Lerner A, Sigal M, Bacalu A et al. A naltrexone double blind placebo controlled study in Israel. Isr J Psychiatry Relat Sci 1992; 29 (1): $36-43$

${ }^{30}$ Nowak M. Naltrexon - der Erfahrungsbericht mit einem Opiat-Langzeitantagonisten. Int. Nemexin ${ }^{\circledR}$ (Naltrexon HCl) Symp. Basel, 1994: $25-30$ 
${ }^{31}$ San L, Pomarol G, Peri JM et al. Follow-up after six months maintenance period on naltrexone versus placebo in heroin addicts. Br J Addict 1991; 86: 190, 983-990

32 Shufman EN, Porat S, Witztum E et al. The efficacy of naltrexone in preventing reabuse of heroin after detoxification. Biol Psychiatry 1994; 35: 935 - 945

${ }^{33}$ Kleber HD, Kosten TR. Naltrexone Induction: Psychologic and Pharmacologic Strategies. J Clin Psychiatry 1984; 45 (9, Sec. 2): 29-38

${ }^{34}$ Kleber HD. Naltrexone. Journal of Substance Abuse Treatment 1985; 2 : $117-122$

${ }^{35}$ Kosten TR, Kleber HD. Strategies to Improve Compliance with Narcotic Antagonists. AM J Drug Alcohol Abuse 1984; 10 (2): 249-266

${ }^{36}$ Anton RF, Hogan I, Jalali B et al. Multiple family therapy and naltrexone in the treatment of opiate dependence. Drug and Alcohol Dependence $1981 ; 8: 157-168$
${ }^{37}$ Koc J, Poser W. Ambulante Abstinenz-Therapie der Opiatabhängigkeit mit Naltrexon. Suchtmedizinisches Symposium der Deutschen Gesellschaft für Drogen- und Suchtmedizin e.V. (DGDS). Vortrag 9. März 1996

${ }^{38}$ Ling W, Wesson DR. Naltrexone treatment for addicted health-care professionals: A collaborative private practice experience. J Clin Psychiatry 1984; 45 : $46-48$

${ }^{39}$ Neto D. Sequential Combined Treatment of Heroin Addicted Patients in Eur Addict Res 1997; 3: 138-145

${ }^{40}$ Roth AS, Ostroff RB, Hoffman RE. Naltrexone as a treatment for repetitive self-injurious behaviour: an open-label trial. J Clin Psychiatry 1996; 57(6): $233-237$

${ }^{41}$ Washton AM, Pottash AC, Gold MS. Naltrexone in addicted business executives and physicians. J Clin Psychiatry 1984; 45: 39-41 